\title{
Pediatric isolated intraparenchymal cerebellar Rosai-Dorfman disease
}

\author{
Bing Wang*1, Janet M. Bruner ${ }^{2}$, Leena Ketonen ${ }^{1}$ \\ ${ }^{1}$ Department of Diagnostic Radiology, The University of Texas MD Anderson Cancer Center, Houston, TX, United States \\ ${ }^{2}$ Department of Pathology, The University of Texas M.D Anderson Cancer Center, Houston, TX, United States
}

Received: April 23, 2017

DOI: $10.5430 /$ ijdi.v4n2p80
Accepted: August 11, 2017

Online Published: August 22, 2017

\begin{abstract}
We illustrate the imaging findings of a case of isolated intraparenchymal cerebellar Rosai-Dorfman disease (RDD) in a child, which to our knowledge is the first reported case of such lesion in a child.
\end{abstract}

Key Words: Rosai-Dorfman disease, Intraparenchymal, Cerebellar, Pediatric, Child

\section{INTRODUCTION}

Rosai-Dorfman disease (RDD) was first described in 1969 as a benign proliferative lesion with systemic symptoms and lymphadenopathy. ${ }^{[1]}$ The disease affects mostly children and young adults with a slight male predilection. ${ }^{[2]}$ While RDD often involves lymph nodes, it can have extranodal involvement. Isolated intracranial RDD is extremely rare. ${ }^{[3]}$ It is especially rare in children where there are only about 10 cases reported in the literature. ${ }^{[4-13]}$ When lesions are intracranial, they are usually extra-axial with dura involvement, not in the brain parenchyma. ${ }^{[14]}$ Isolated intracranial RDD without dural attachment is extremely rare. ${ }^{[15]}$ We found only a few reported cases in the literature of isolated intraparenchymal RDD involving the cerebellum, all cases are in adults, even though the disease predominantly affects children and young adults. ${ }^{[16]}$ We present here a case of RDD isolated to the brain parenchyma of the cerebellum in a child, which to our knowledge has not been reported in the literature.

\section{CASe PResentation}

The patient is a 14-year-old right-handed boy without significant past medical history who noted a sudden headache in the back of his head while watching TV. When the symptoms became more severe he went to a local emergency room. He underwent blood and urine tests and was sent home, because the tests were within normal limits. Two days later he had severe headaches again. He saw a physician and received injection of pain medication which helped the pain.

A week later, he underwent a CT scan, followed by an MRI. The imaging studies showed a right cerebellar lesion. After discussion with family, the decision was made to surgically resect the lesion, both for treatment of the lesion and for pathologic diagnosis. The patient underwent a suboccipital craniotomy, and the lesion was resected without complication. The most recent follow up MRI showed gross total resection of the cerebellar lesion with no evidence of recurrence. Patient returned to normal life and had no residual symptoms during his last clinical follow up 2 years later.

\footnotetext{
*Correspondence: Bing Wang; Email: bing.wang@mdanderson.org; Address: Department of Diagnostic Radiology, The University of Texas MD Anderson Cancer Center, Houston, TX, United States.
} 


\section{IMAGING FINDINGS}

CT showed the presence of a hyperdense intraparenchymal lesion in the right cerebellar hemisphere, with surrounding with edema (see Figure 1A).

MRI demonstrated a solitary $3.0 \mathrm{~cm} \times 2.6 \mathrm{~cm}$ homogeneously enhancing lesion in the right cerebellar hemisphere (see Figure 1B). On the T2 FLAIR image, the lesion was slightly low in signal compared with cerebellum with sur- rounding edema (see Figure 1C). On the T2 gradient echo image (GRE), a tiny focus of susceptibility signal void is present anteriorly, which could represent a tiny focus of hemorrhage (see Figure 1D). On the T1 weighted image, the lesion is isointense to the brain tissue (see Figure 1E). On the diffusion image (DWI), there is no evidence of restricted diffusion (see Figure 1F). There is no displacement of the fourth ventricle or hydrocephalus.

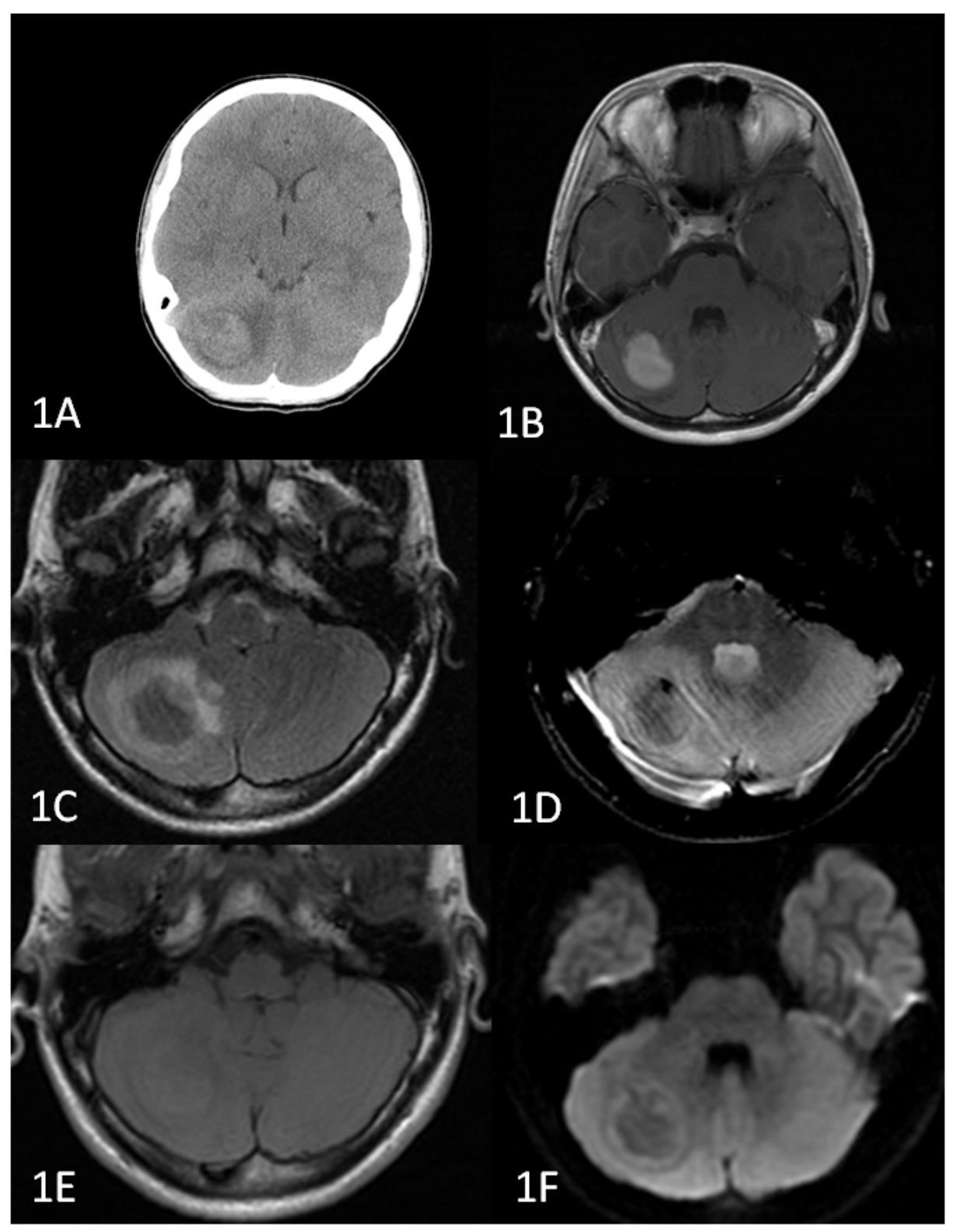

Figure 1. 1A: Noncontrast $\mathrm{CT}$ demonstrates a hyperdense intraparenchymal lesion in the right cerebellar hemisphere, with surrounding hypodensity compatible with edema. 1B: Post contrast T1 image shows a solitary $3.0 \mathrm{~cm} \times 2.6 \mathrm{~cm}$ homogeneously enhancing lesion in the right cerebellar hemisphere away from the 4th ventricle. 1C: On T2 FLAIR image, the lesion is slightly low in signal compared with cerebellum; the hyperintensity surrounding the lesion is most consistent with edema. 1D: On T2 GRE, a tiny focus of susceptibility signal void anteriorly could represent a tiny focus of hemorrhage. 1E: On T1, the lesion is isointense to the brain tissue. 1F: On DWI, there is no evidence of restricted diffusion. 


\section{Pathological findings}

Gross pathology shows an irregular ovoid resection of cerebellum measuring $3.5 \mathrm{~cm} \times 2.0 \mathrm{~cm} \times 1.5 \mathrm{~cm}$. The specimen is firm. The fragments of cerebellum attached to the external surface are gray-white and soft. The normal foliar pattern is noted focally. Within the specimen is a firm, yellow-tan tumor which is well-circumscribed. It measures up to $3.0 \mathrm{~cm}$ $\times 2.0 \mathrm{~cm}$. In the center of the tumor is an area of stellate gray-white discoloration.

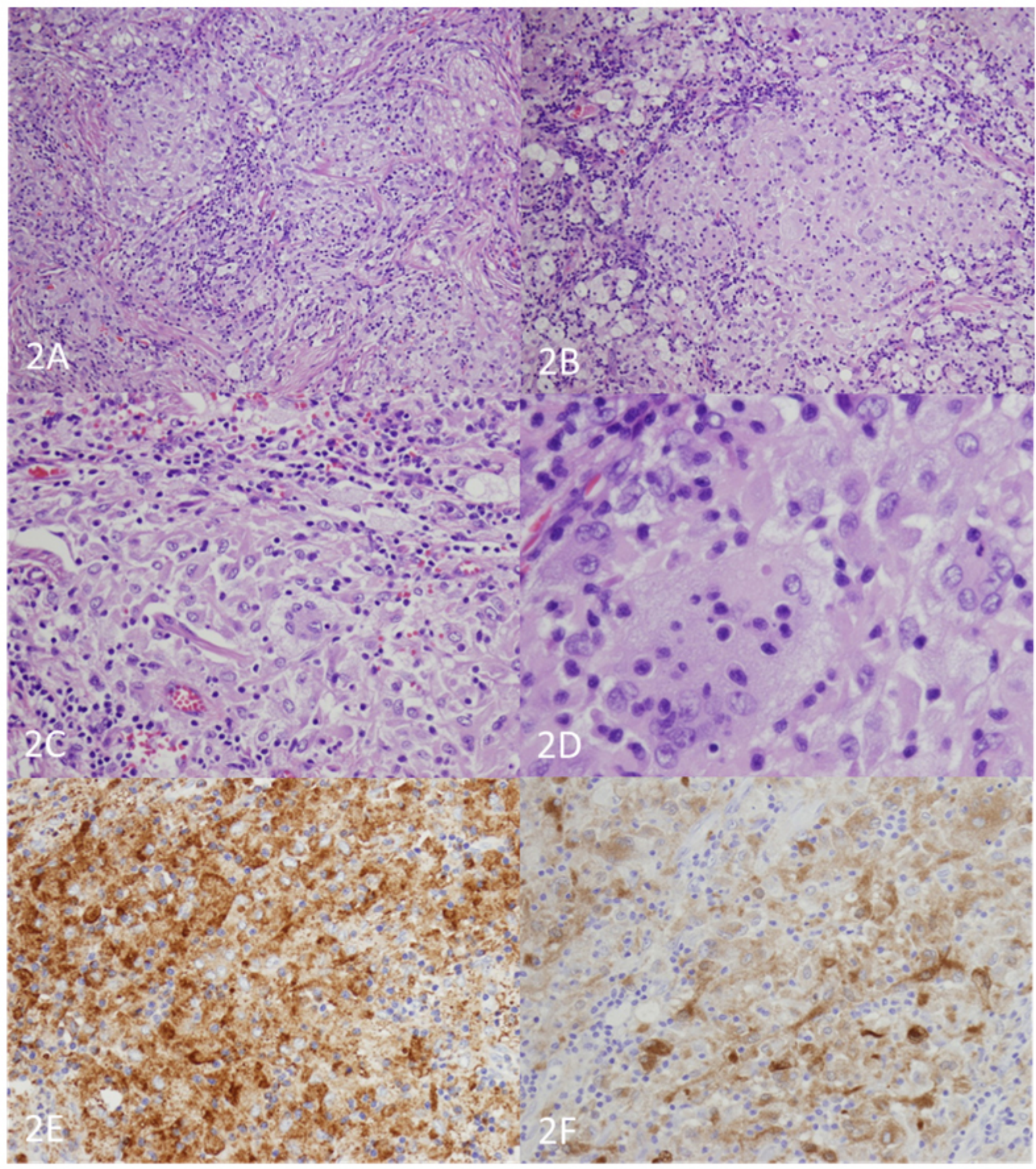

Figure 2. 2A: Histological section (H\&E) shows lobules of histiocytes with pale granular cytoplasm, surrounded by mononuclear inflammatory cells. 2B: Histological section (H\&E) shows multinucleated giant cells, foamy histiocytes, lymphocytes, plasma cells, and Touton-like giant cells. 2C: Histological section (H\&E) shows giant cells, histiocytes, and mononuclear cells. 2D: Histological section (H\&E) shows scattered lymphocytes within the cytoplasm of histiocytes (emperipolesis). 2E: Immunohistochemistry shows CD68 antibody+. 2F: Immunohistochemistry shows S-100 antibody+. 
Histologic sections show the classic appearance of RosaiDorfman disease with lobular aggregates of histiocytes surrounded by thin connective tissue septae containing mature lymphocytes. There are nodules of granulomatous-type noncaseating lymphohistiocytic inflammation. Some of the histiocytes are multinucleated. Within the cytoplasm of histiocytes, scattered lymphocytes are noted (emperipolesis). The adjacent cerebellum shows Rosenthal fiber formation and reactive gliosis (see Figures 2A-2D).

Immunohistochemistry shows strong diffuse reactivity in the histiocytes for CD68 (see Figure 2E) and focal strong reactivity for S-100 (see Figure 2F) protein. A stain for organisms (GMS) is negative.

\section{Discussion}

RDD disease, sinus histiocytosis with massive lymphadenopathy, rarely involves the intracranial structures without other sites of involvement. When it does, it commonly is dural-based, involving the frontal and parietal regions and the skull base. Extranodal disease usually involves eyes, salivary glands, thyroid and upper respiratory tract rather than brain. ${ }^{[2]}$ Isolated intraparenchymal Rosai-Dorfman disease is extremely rare.

This case is unique because it only involved cerebellum without nodal involvement, which has not been described before in children. Although the disease is characterized by sinus histiocytosis and massive lymphadenopathy, our case does not have lymphadenopathy; his full lymph node imaging search was negative. His presentation is limited to brain parenchyma, not just intracranial space. We found only one other reported case of isolated intracranial parenchymal involvement in a child, which involved supratentorial thalamus and insula. ${ }^{[12]}$ We could not find another similar reported case of isolated intraparenchymal involvement in the infratentorial compartment in a child, as in our case.

This lesion is highly cellular on pathology, which explains why it is slightly hyperdense on CT and slightly hypointense on the MRI T2 and FLAIR sequences. A range of low to hyperintensity on $\mathrm{T} 2$ sequence has been described. In our case, gross total surgical resection resulted in no recurrence on follow up.

\section{SUMMARY}

To our knowledge, this case is the first reported case of isolated intraparenchymal cerebellar Rosai-Dorfman disease in a child. Despite of its rarity, physicians should be aware of this entity and take it into consideration when treating solitary cerebellar lesions even in children.

\section{Conflicts of InTERest Disclosure}

The authors have no conflict of interest related to this publication.

\section{REFERENCES}

[1] Rosai J, Dorfman RF. Sinus histiocytosis with massive lymphadenopathy: a newly recognized benign clinicopathological entity. Arch Pathol. 1969; 87: 63-70 . PMid:5782438

[2] Raslan OA, Schellingerhout D, Fuller GN, et al. Rosai-Dorfman disease in neuroradiology: imaging findings in a series of 10 patients. AJR Am J Roentgenol. 2011 Feb; 196(2): W187-93. PMid:21257861 https://doi.org/10.2214/AJR.10.4778

[3] Konishi E, Ibayashi N, Yamamoto S, et al. Isolated intracranial RosaiDorfman disease (sinus histiocytosis with massive lymphadenopathy). AJNR Am J Neuroradiol. 2003; 24: 515-518. PMid:12637307

[4] Di Rocco F, Garnett MR, Puget S, et al. Cerebral localization of Rosai-Dorfman disease in a child. Case report. J Neurosurg. 2007; 107: 147-151. PMid:18459887

[5] Griffiths SJ, Tang W, Parameswaran R, et al. Isolated intracranial Rosai-Dorfman disease mimicking meningioma in a child. Br J Neurosurg. 2004; 18: 293-297. PMid:15327236 https : //doi .org/10 $.1080 / 02688690410001732788$

[6] Gupta DK, Suri A, Mahapatra AK, et al. Intracranial Rosai-Dorfman disease in a child mimicking bilateral giant petroclival meningiomas: a case report and review of literature. Childs Nerv Syst. 2006; 22: 1194-1200. PMid:16552567 https ://doi .org/10.1007/s0 0381-006-0055-1

[7] Gupta K, Bagdi N, Sunitha P, et al. Isolated intracranial RosaiDorfman disease mimicking meningioma in a child: a case report and review of the literature. Br J Radiol. 2011; 84: e138-e141. https://doi.org/10.1259/bjr/15772106

[8] Lungren MP, Petrella JR, Cummings TJ, et al. Isolated intracranial Rosai-Dorfman disease in a child. AJNR Am J Neuroradiol. 2009; 30: E148-E149. https://doi.org/10.3174/ajnr.A1812

[9] Shaver EG, Rebsamen SL, Yachnis AT, et al. Isolated extranodal intracranial sinus histiocytosis in a 5-year-old boy. Case report. J Neurosurg. 1993; 79: 769-773. PMid:8410258 https://doi .org/ 10.3171/jns.1993.79.5.0769

[10] Woodcock RJ Jr, Mandell JW, Lipper MH. Sinus histiocytosis (Rosai-Dorfman disease) of the suprasellar region: MR imaging findings—a case report. Radiology. 1999; 213: 808-810 PMid:10580957 https://doi.org/10.1148/radiology.213.3.r99dc30808

[11] Yang X, Yu C, Li K, et al. Isolated intracranial Rosai-Dorfman disease: a case report with $\mathrm{CT}$ and MR findings. Eur J Radiol Extra. 2007; 61: 77-80 https://doi.org/10.1016/j.ejrex.2007.0 1.001

[12] Miletic H, Rohling R, Stenzel W, et al. 8-year-old child with a lesion in the left nucleus lentiformis. Brain Pathol. 2008; 18: 598-601. PMid:18782173 https://doi .org/10.1111/j.1750-3639.20 $08.00209 \cdot \mathrm{x}$

Published by Sciedu Press 
[13] Maratos E, Bridges R, MacKinnon A, et al. Isolated intracranial Rosai-Dorfman disease in a child, a case report and review of the literature. Child's Nervous System. 2014; 30(9): 1595-1600. PMid:24864019 https://doi .org/10.1007/s00381-014-243 7-0

[14] Andriko JA, Morrison A, Colegial CH, et al. Rosai-Dorfman disease isolated to the central nervous system: a report of 11 cases. Mod Pathol. 2011; 14: 172-178 PMid:11266522 https://doi.org/10 $.1038 /$ modpathol .3880278
[15] Fukushima T, Yachi K, Ogino A, et al. Isolated intracranial RosaiDorfman disease without dural attachment-case report. Neurol Med Chir (Tokyo). 2011; 51: 136-140. https://doi.org/10.2176/ nmc. 51.136

[16] Beros V, Houra K, Rotim K, et al. Isolated cerebellar intraparenchymal Rosai-Dorfman disease-case report and review of literature. Br J Neurosurg. 2011 Apr; 25(2): 292-6. PMid:21344967 https : //doi.org/10.3109/02688697.2010.546899 